Gefässchirurgie $2020 \cdot 25: 345-351$ https://doi.org/10.1007/s00772-020-00670-z Online publiziert: 4. August 2020

(C) Der/die Autor(en) 2020
M. Kaschwich • F. von Haxthausen ${ }^{2}$ T. Aust ${ }^{2}$ F. Ernst ${ }^{2}$ - M. Kleemann ${ }^{1}$

${ }^{1}$ Klinik für Chirurgie, Bereich Gefäßchirurgie, Universitätsklinikum Schleswig-Holstein, Campus Lübeck, Lübeck, Deutschland

${ }^{2}$ Institut für Robotik und kognitive Systeme, Universität zu Lübeck, Lübeck, Deutschland

\title{
Roboterbasierte Ultraschallsteuerung
}

\section{Pilotstudie zur Roboter-assistierten Ultraschall-gesteuerten peripheren endovaskulären Intervention am Phantom - das RoGUS-PAD-Projekt}

\section{Einleitung}

\begin{abstract}
„Nichts auf der Welt ist so kraftvoll wie eine Idee, deren Zeit gekommen ist" (Zitat: Victor Hugo). Unter Berücksichtigung des technischen Fortschritts sind automatisierte Assistenzsysteme in der endovaskulären Chirurgie heutzutage vorstellbar. Die Autoren beschreiben in diesem Artikel ein ambitioniertes Projekt, in dem ein roboterbasiertes ultraschallgesteuertes Assistenzsystem als Alternative zur konventionellen Verwendung von Röntgenstrahlen und Röntgenkontrastmittel im Rahmen von peripheren endovaskulären Interventionen dienen soll.
\end{abstract}

\section{Kooperation}

Die Herstellung des ultraschallfähigen Phantoms erfolgte in Zusammenarbeit mit der Firma HumanX GmbH, Freiheitsstr. 124/126, 15745 Wildau, Deutschland.

\section{Patentanmeldung}

Für das beschriebene Projekt erfolgte eine Patentanmeldung der Universität zu Lübeck beim Deutschen Patent- und Markenamt (DPMA). Anmeldenummer: DE 102020109 593.7.

\section{Anmerkung}

Die Ergebnisse der Pilotstudie wurden auf der AUTOMED - Automation in Medical Engineering 2020 im Rahmen eines Kongresspapers [9] vorgestellt.

\section{Das ungelöste Problem endovaskulärer Techniken}

Für die Navigation der Instrumente im Rahmen endovaskulärer Interventionen ist noch immer die Anwendung von Röntgenstrahlen notwendig. Als Folge ist die Strahlenbelastung, insbesondere für den Interventionalisten, zum Teil immer noch sehr hoch [8]. Darüber hinaus werden Röntgenkontrastmittel für die intraprozedurale Darstellung der Gefäße benötigt. Ihr Einsatz birgt jedoch das Risiko einer kontrastmittelbedingten Nierenschädigung [20]. Die Entwicklung alternativer Navigationsmethoden ist daher von großer Relevanz [13].

\section{Intraprozedurale Ultraschall-} bildgebung als Alternative zur Angiographie mit Röntgenstrahlen

Einer der aussichtsreichsten Ansätze zur Verminderung der Strahlenexposition bei peripheren endovaskulären Interventionen besteht im vermehrten Einsatz der Ultraschallbildgebung [2, $3,5]$. Dieses Verfahren ist frei von ionisierender Strahlung und erlaubt eine schnelle Visualisierung und Beurteilung der anatomischen Strukturen. Darüber hinaus liefert die Duplexsonographie dem Untersucher zusätzliche Informationen über die Strömungskinetik des Blutes, welche für die Beurteilung hämodynamisch relevanter Stenosen wichtig sind [22]. Die Duplexsonographie hat sich daher zum bevorzugten Instrument insbesondere in der Diagnostik und Nachsorge vaskulärer Pathologien entwickelt [1]. Des Weiteren wurde bereits in einem klinischen Setting gezeigt, dass in Ausnahmefällen periphere endovaskuläre Eingriffe, die normalerweise unter Röntgendurchleuchtung erfolgen, auch duplexsonographisch gestützt mit manueller Schallkopfführung durchführbar sind $[2,3,5]$. Auch in den Leitlinien der European Federation of Societies for Ultrasound in Medicine and Biology (EFSUMB) wird die Möglichkeit einer ultraschallgestützten endovaskulären Intervention erwähnt [7]. Nicht zuletzt nimmt die Qualität der Ultraschalluntersuchung durch technische Neuerungen wie verbesserte Bildgebungstechniken und automatisierte Bildbearbeitung [6, 10] stetig zu.

\section{Das Problem der manuellen Schallkopfführung}

Während einer peripheren endovaskulären Intervention ist eine manuelle Führung des Schallkopfes durch den Operateur oder entsprechend geschultes assistierendes Personal schwer umsetzbar und daher wenig praktikabel. Es ist dem Arzt z. B. nicht möglich, parallel zur Intervention den Ultraschallkopf zu steuern, da beide Hände zur Durchführung der Intervention benötigt werden. Zur Lösung 


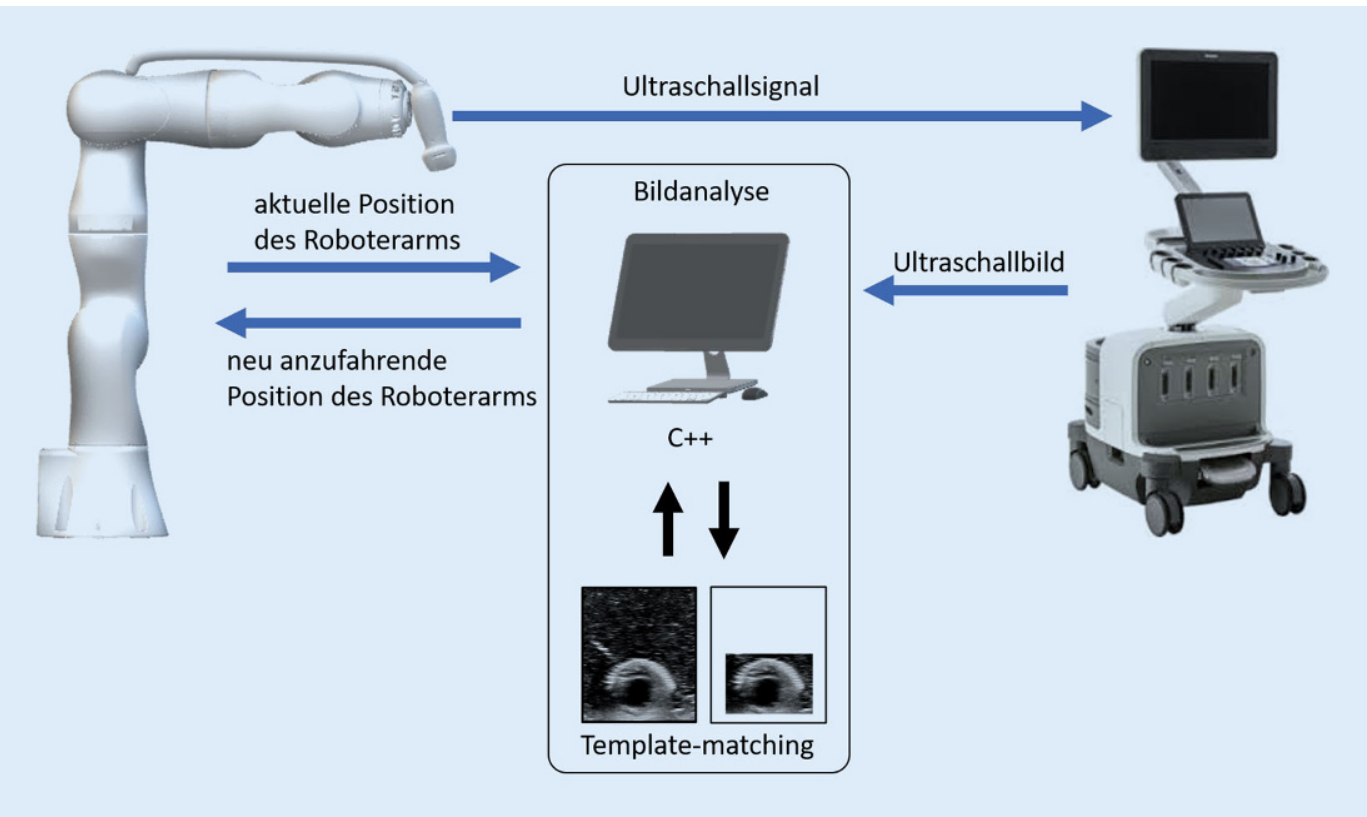

Abb. $1 \triangleleft$ Zusammenhang zwischen den einzelnen Komponenten des Systems. Mit freundlicher Genehmigung, (c) M. Kaschwich, M. Kleemann. Alle Rechte vorbehalten

dieses Problems bietet sich eine roboterbasierte Steuerung des Ultraschallkopfes an. Ein Roboter ist in der Lage, bestimmte Positionen mit hoher Präzision anzufahren und kann Untersuchungsschritte mehrmals wiederholen, ohne dabei zu ermüden $[15,26]$.

\section{Motivation und Ziel des Projektes}

Primäres Ziel des RoGUS-PAD (RoboticGuided Ultrasound System for Peripheral Arterial Disease)-Projektes ist (1) die Durchführung peripherer endovaskulärer Interventionen ohne Verwendung von Röntgenstrahlung und Röntgenkontrastmittel sowie (2) die Verbesserung der intraprozeduralen Echtzeitvisualisierung. Dies soll durch ein neuartiges roboterbasiertes ultraschallgesteuertes Assistenzsystem erreicht werden.

\section{Material und Methoden}

\section{Forschungsumfeld}

Zur Umsetzung eines solchen Projekts wird ein interdisziplinäres Forschungsund Entwicklungsumfeld und -team benötigt, das die Expertise aus Gefäßmedizin und Robotik vereint. Das RoGUSPAD-Projekt ist ein Gemeinschaftsprojekt der Klinik für Chirurgie, Bereich für Gefäßchirurgie des Universitätsklinikums Schleswig-Holstein (UKSH), Cam- pus Lübeck und dem Institut für Robotik und kognitive Systeme der Universität zu Lübeck.

\section{Robotersystem}

Für die Versuche wurde ein KUKA-Roboterarm-System (LBR iiwa 7 R800, KUKA, Augsburg, Deutschland) verwendet (• Abb. 2a-d). Hierbei handelt es sich um einen sogenannten kollaborativen Roboterarm mit 7 Freiheitsgraden. Der Roboterarm ist zertifiziert als „robotische Komponente zur Integration in ein Medizinprodukt“. Dieses System wurde bereits erfolgreich für ähnliche Projekte eingesetzt $[23,27]$.

\section{Ultraschallsystem}

Für den Ultraschallscan verwendeten wir ein 2-D-Lineartastkopf (L12-3, Philips Healthcare, Best, Niederlande) mit einem speziell angefertigten Tastkopfhalter, der am Endeffektor des Roboterarms montiert wurde. Eine Softwareschnittstelle ermöglichte das Echtzeit-Streaming von 8-Bit-Graustufenbildern des Ultraschalls von der Ultraschallstation (EPIQ 7, Philips Healthcare, Best, Niederlande) auf einen Computer. Zusätzlich erlaubte eine speziell angefertigte Middleware, Befehle vom Computer an den Roboterarm zu senden. Ein C++-Programm, führte die Bildverarbeitung durch und sendete die resultierenden Befehle zur Neupositionierung der Ultraschallsonde am Endeffektor (• Abb. 1).

\section{Ultraschallfähiges Phantom}

Vor dem Hintergrund des Deutschen Tierschutzgesetzes ( $\$ 7$ TierSchG) und den EU Guidelines for the Protection of Animals (2 and 3 86/609/EEC), soweit wie möglich auf Tierversuche zu verzichten, wurde speziell für dieses Projekt gemäß der „3R“-Regel („,replace, reduce, refine“) ein ultraschallfähiges Phantom mit austauschbaren patientenspezifischen 3-D-gedruckten Gefäßpathologien entwickelt, an dem die erste Pilotstudie durchgeführt wurde (• Abb. 2a-d). Die Methodik und iterative Erstellung des Gefäßphantoms ist einer aktuellen Publikation unserer Arbeitsgruppe zu entnehmen [12].

\section{Ergebnisse der Pilotstudie}

\section{Semiautomatischer 2-D- Ultraschallscan}

$\mathrm{Zu}$ Evaluation der technischen Umsetzbarkeit des Systems wurde zunächst ein semiautomatischer 2-D-UltraschallScan am Simulator entwickelt.

Der Ablauf des Scanvorgangs ist wie folgt: 
1. Mit einem speziell entwickelten Handführungsmodus [16] platziert der Untersucher die am Roboterarm befestigte Ultraschallsonde so, dass im Ultraschallbild ein Querschnittsbild der Arterie sichtbar ist (- Abb. 2a).

2. Innerhalb des gestreamten Ultraschallbilds kann der Untersucher den Bereich auswählen, der von Interesse ist, nämlich das Template, welches die Arterie enthält (• Abb. 2a).

3. Auf den nachfolgenden Bildern wird ein Templatevergleich durchgeführt, um die Arterie im Bild zu finden und anschließend nach distal zu scannen (-Abb. 2b).

4. Nach dem Scannen der Arterie (- Abb. 2c) kehrt der Roboterarm in die Ausgangsstellung zurück (•Abb. 2d).

\section{Evaluierung der Robotersteuerung}

Zur Evaluation der Robotersteuerung wurden insgesamt 30 Scandurchläufe in verschiedenen Phantompositionen $\left(0^{\circ}, 30^{\circ},-30\right)$ durchgeführt. In 27 von 30 Durchläufen wurde die Phantomarterie (3-D-gedruckte A. femoralis) erfolgreich gescannt. In einem Versuch erreichte der Roboter das Ende seines Arbeitsbereichs, in zwei Versuchen lief der Roboterarm in eine Singularität und konnte den Scanvorgang nicht beenden (•Abb. 3).

\section{Diskussion}

Die Ergebnisse unserer ersten Pilotversuche am Phantom bestätigten unsere Hypothese, dass die Entwicklung eines Roboterassistenzsystems für ultraschallgesteuerte periphere endovaskuläre Interventionen mit dem von uns genutzten Robotersystem technisch umsetzbar ist.

\section{) Roboterassistenzsysteme für ultraschallgesteuerte Interventionen sind umsetzbar}

Nach unserem Kenntnisstand ist dies die erste Studie, die für periphere endovaskuläre Eingriffe ein robotisches Ultraschallassistenzsystem beinhaltet.

Gefässchirurgie 2020 -25:345-351 https://doi.org/10.1007/s00772-020-00670-z

(c) Der/die Autor(en) 2020

\section{Kaschwich · F. von Haxthausen · T. Aust · F. Ernst · M. Kleemann}

Roboterbasierte Ultraschallsteuerung. Pilotstudie zur Roboterassistierten Ultraschall-gesteuerten peripheren endovaskulären Intervention am Phantom - das RoGUS-PAD-Projekt

Zusammenfassung

Hintergrund. Die periphere endovaskuläre Chirurgie ist nach wie vor durch die Anwendung von Röntgenstrahlen und Röntgenkontrastmittel für die intraprozedurale Navigation der Instrumentarien ein Verfahren mit potenziellen Risiken und Nebenwirkungen.

Projektziel. Ziel des RoGUS-PAD (RoboticGuided Ultrasound System for Peripheral Arterial Disease)-Projektes ist die Entwicklung eines roboterbasierten ultraschallgesteuerten Assistenzsystems für periphere endovaskuläre Interventionen zur Verringerung und ggf. Vermeidung von Röntgenstrahlung und Röntgenkontrastmittel sowie Verbesserung der Echtzeitvisualisierung.

Material und Methoden. Für die Bildgebung wurde ein 2-D-Ultraschall-Lineartastkopf (L12-3, Philips Healthcare, Best, Niederlande) am Endeffektor eines Roboterarms (LBR iiwa 7 R800, KUKA, Augsburg, Deutschland) montiert. Die ersten Versuche wurden an einem eigens für dieses Projekt entwickelten ultraschallfähigen Phantom durchgeführt. Die Bildverarbeitung und Robotersteuerung erfolgten durch ein speziell entwickeltes Programm in $\mathrm{C}++$.

Ergebnisse. Zur Testung der technischen Umsetzbarkeit des Projektes konnten wir einen semiautomatischen 2-D-Ultraschallscan einer peripheren Arterie am Phantom durchführen. In 27 von 30 Durchläufen zeigte sich ein erfolgreicher Scanvorgang.

Schlussfolgerung. Unsere ersten Ergebnisse bestätigten, dass die Entwicklung eines roboterbasierten Assistenzsystems für ultraschallgesteuerte periphere endovaskuläre Interventionen technisch umsetzbar ist. Dies stützt unsere Ambitionen einer Translation des Systems in die tägliche klinische Praxis.

\section{Schlüsselwörter}

Roboterassistenz $\cdot$ Roboterbasierter Ultraschall · Endovaskuläre Interventionen . Ultraschallgestützte Intervention · Phantom

\section{Robot-based ultrasound guidance. Pilot study on robot-assisted ultrasound-guided peripheral endovascular intervention on a phantom-The RoGUS-PAD project}

\section{Abstract}

Background. Peripheral endovascular surgery is still a procedure with potential risks and side effects due to the use of $X$-rays and contrast agents for the intraprocedural navigation of instruments.

Objective. The aim of the robotic-guided ultrasound system for peripheral arterial disease (RoGUS-PAD) project is the development of a robot-based ultrasound-guided assistance system for peripheral endovascular interventions to reduce or even avoid X-rays and contrast agents as well as improve realtime visualization.

Material and methods. For imaging purposes, a 2D ultrasound linear probe (L12-3, Philips Healthcare, Best, The Netherlands) was mounted on the end effector of a robotic arm (LBR iiwa 7 R800, KUKA, Augsburg, Germany). The first tests were performed on an ultrasound-capable phantom developed especially for this project. The image processing and robot control were carried out by a specially developed program in $\mathrm{C}++$. Results. To evaluate the technical feasibility of the project we were able to perform a semiautomatic $2 \mathrm{D}$ ultrasound scan of a peripheral artery on the phantom. In 27 out of 30 runs a scan was successfully performed. Conclusion. The initial results confirmed that the development of a robot-based assistance system for ultrasound-guided peripheral endovascular interventions is technically feasible. This supports our ambitions for the translation of the system into daily clinical practice.

\section{Keywords}

Robot assistance - Robot-guided ultrasound . Endovascular interventions - Ultrasoundguided intervention - Phantom 

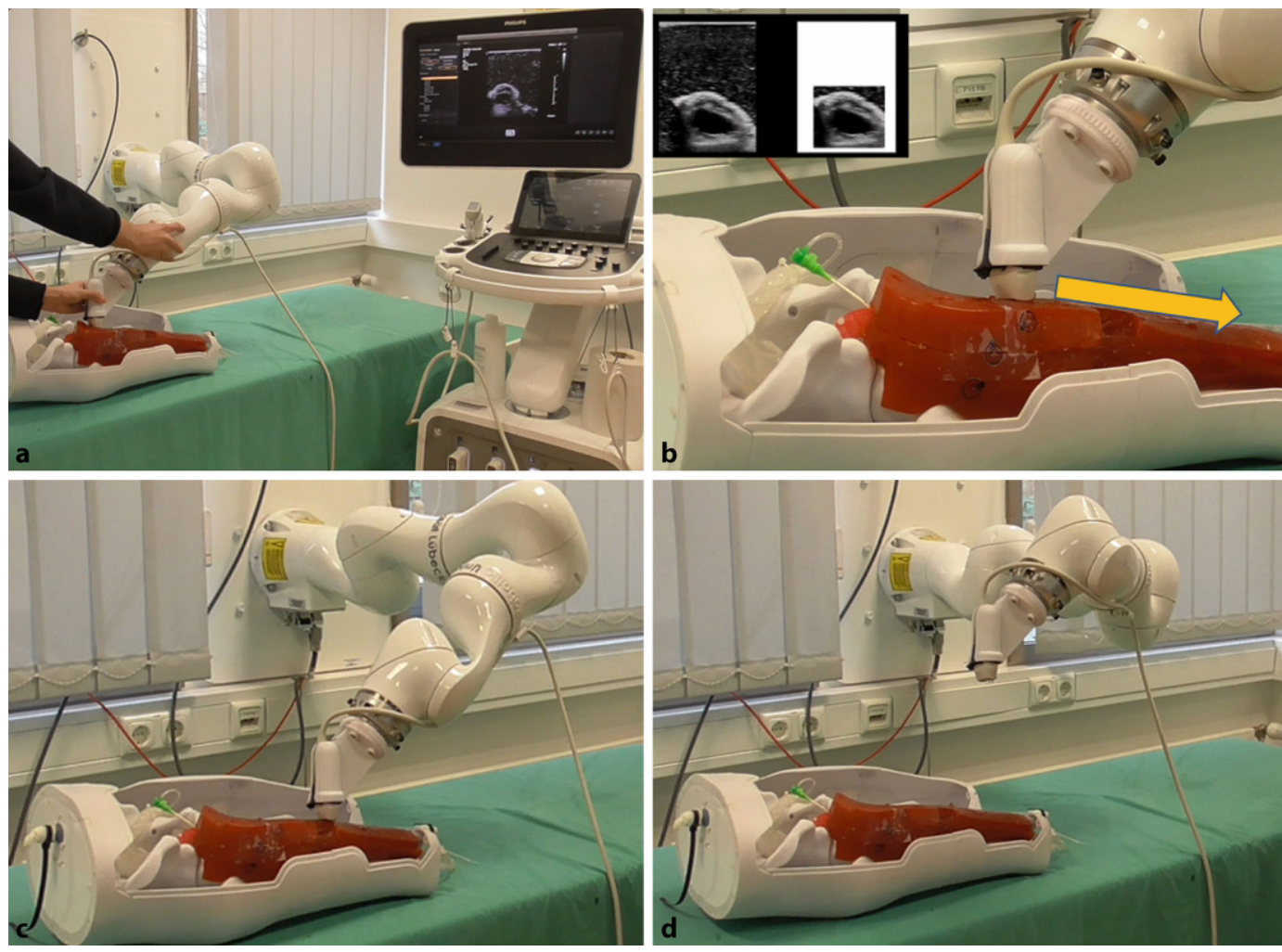

Abb. $2<$ Semiautomatischer 2-D-Scan am Simulator: a Manuelle Positionierung des Ultraschallkopfes. b Automatischer Scan des Phantomgefäßes durch Bildabgleich (kleines Bild links oben). c Fortgeführter automatischer Scan. d Rückkehr des Roboterarms in die Ausgangsposition nach Beendigung des Scanvorgangs. Mit freundlicher Genehmigung, $\odot$ M. Kaschwich, M. Kleemann. Alle Rechte vorbehalten

Die Idee einer robotergestützten Ultraschalldiagnostik ist allerdings nicht neu. Maturo et al. entwickelten bereits in den 1980er Jahren eine automatisierte Ultraschalluntersuchung der weiblichen Brust [17]. Abolmaesumi et al. [4] untersuchten den Einsatz eines Roboterarms für die Diagnostik der A. carotis. Virga et al. [27] entwickelten ein autonomes Robotersystem für Ultraschalluntersuchungen der Bauchaorta. Eine klinische Studie von Wang et al. [28] beschreibt die Anwendung eines robotergestützten Ultraschalls für fetale Diagnostik. Die zitierten Studien stützen unsere Einschätzung, dass roboterbasierter Ultraschall sowohl technisch als auch klinisch umsetzbar ist. Im Februar 2018 erhielt das Corindus CorPath GRX-RoboterSystem der Fa. Corindus Vascular Robotics Inc. die Freigabe der US Food and Drug Administration (FDA) für periphere Gefäßinterventionen. Dieses System hat einen automatisierten Vorschub der Drähte und Kathetersysteme zu eigen, nutzt jedoch weiterhin konventionelle Angiographiesysteme mit Röntgenstrahlen und Kontrastmittelapplikationen und wurde kürzlich in diesem Journal beschrieben [14]. Allerdings ist bis dato noch kein roboterbasiertes Ultraschallsystem beschrieben, dass den Ansprüchen der täglichen klinische Praxis standhält.

Einer der Hauptgründe hierfür ist sicherlich in der Komplexität einer Ultraschalluntersuchung zu suchen. Eine Ultraschalluntersuchung ist ein hochdynamischer Prozess, bei der der Untersucher nicht nur mit der Anatomie der zu untersuchenden Körperregion gut vertraut sein muss, sondern es ist auch eine stetige Neupositionierung des Schallkopfes notwendig. Des Weiteren ist ein kontinuierlicher und gleichmäßiger Druck des Schallkopfes wichtig, damit der Kontakt zur Haut und damit das Ultraschallsignal nicht unterbrochen wird. Gegebenenfalls müssen auch ungewollte Bewegungen des Patienten kompensiert werden.

Die oben genannten Gründe sind dafür verantwortlich, dass die Qualität einer Ultraschalluntersuchung entscheidend von der Erfahrung und dem Können des Untersuchers abhängt und somit eine hohe Intra- und Inter-BeobachterVariabilität vorhanden ist [25].

Auch Sicherheitsaspekte, Patienten und -Behandlerakzeptanz für robotische
Assistenzsysteme sind $\mathrm{zu}$ respektieren. Eine begleitende Entwicklung ist daher unter den Gesichtspunkten des Medizinproduktegesetztes [30] und Health Technology Assessment (HTA) zu fordern.

Zur Lösung dieser Probleme arbeiten mehrere internationale Forschergruppen an der Automatisierung der Schallkopfführung mithilfe von kollaborativen Robotern [21]. Diese sind dank integrierter Kraftsensorik sicher in direktem Kontakt zum Menschen einsetzbar. Hier werden von aktuellen Robotersystemen insbesondere die technischen und rechtlichen Vorgaben zur Mensch-RoboterKollaboration (DIN EN ISO 10218- 1 und -2) sowie zum Software-Lebenszyklus für medizinische Software (DIN EN ISO 62304) eingehalten. Diverse Systeme zur robotergestützten Ultraschallbildgebung wurden in den letzten Jahren entwickelt, sowohl für diagnostische Zwecke (z. B. Karotis- [19], Beinvenen- [11] oder Leber-Scans [18]) als auch für interventionelle Anwendungsfälle (Nadelpunktionen [29] und Tumorverfolgung [24]). Die Vorteile der automatisierten Schallkopfführung liegen dabei vor allem in der Reproduzierbarkeit der Bilderfas- 

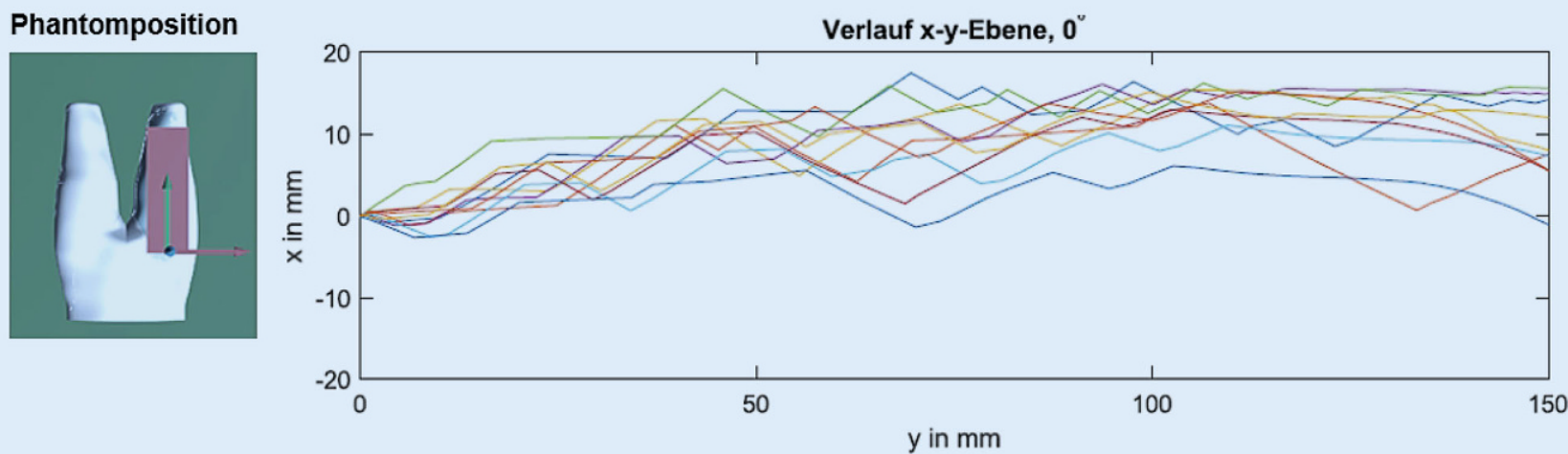

Verlauf $x-y$-Ebene, $0^{\circ}$
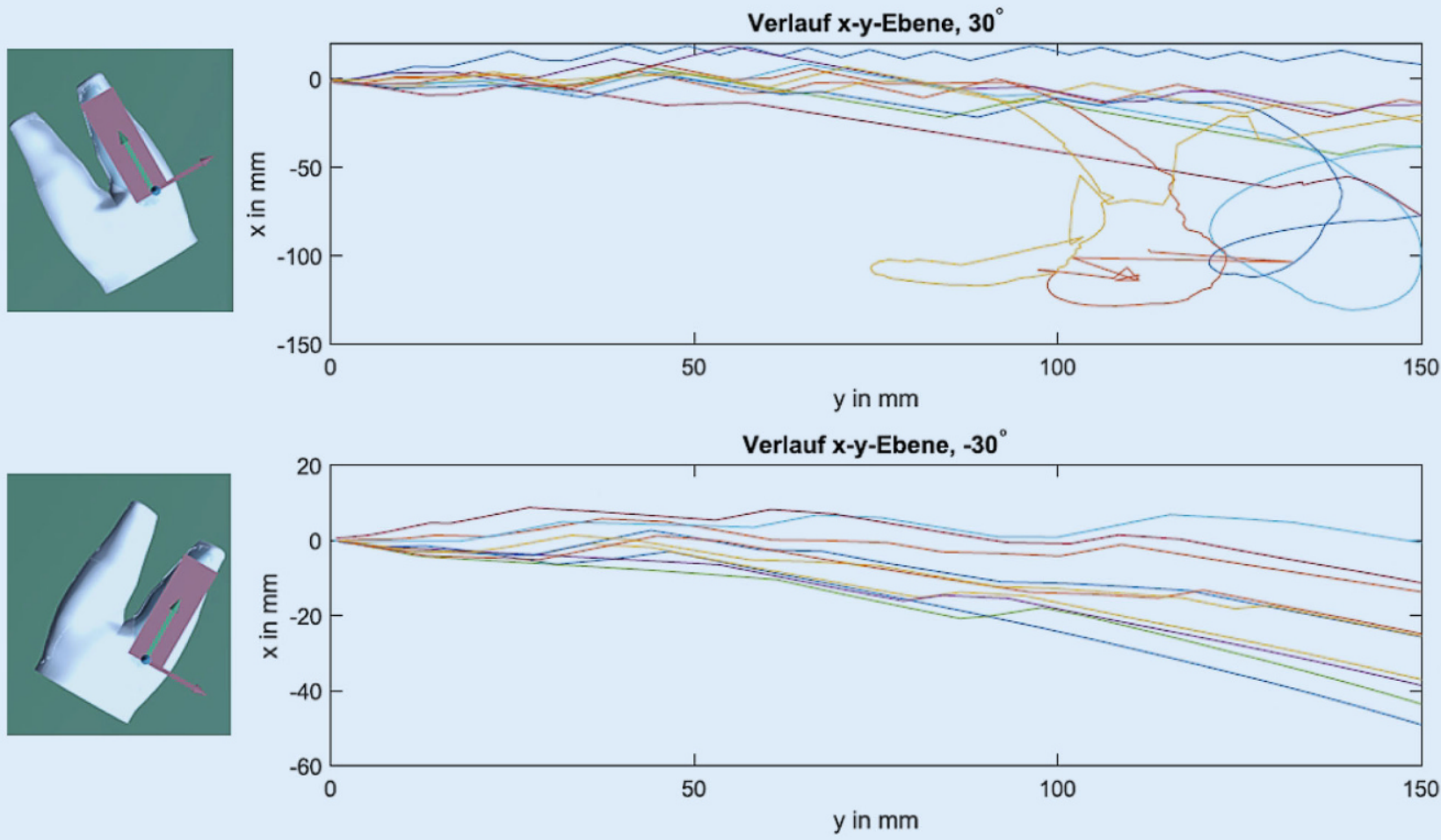

Abb. 3 A Verlauf des Roboterarms in der x-y-Ebene bei unterschiedlicher Position des Phantoms. Pro Position sind 10 Durchläufe dargestellt. Abbildung modifiziert aus Bachelorarbeit von T. Aust. Titel: Visual Servoing für endovaskuläre Eingriffe mittels Ultraschalldaten, Universität zu Lübeck. Mit freundlicher Genehmigung T. Aust

sung hinsichtlich des Andrucks und der Position und der Möglichkeit der automatischen, bildbasierten Nachführung des Schallkopfes bei Bewegungen des Zielgebiets.

\section{》) Das RoGUS-PAD-Projekt ist ein Assistenzsystem zu Unterstützung des Arztes}

Ob sich ein solches System in den klinischen Alltag integrieren lässt, hängt außerdem von der Akzeptanz der Anwender und nicht zuletzt von der des Patien- ten ab. In diesem Zusammenhang konnten wir in einer internationalen Umfrage (unveröffentlichte Daten) zeigen, dass die Akzeptanz einer solchen Technologie von einem großen Teil der potenziellen Anwender getragen wird.

Dennoch ist davon auszugehen, dass einige Ärzte die Angst hegen, durch einen Roboter ersetzt zu werden. Es sei hier noch einmal erwähnt, dass wir mit dem RoGUS-PAD-Projekt ein Assistenzsystem entwickeln wollen, dass den Arzt optimal autonom während der Intervention unterstützt - es ist nicht das Ziel, den Arzt zu ersetzen. Dieser behält stets die Kontrolle über den Eingriff.

Es ist davon auszugehen, dass mit dem zunehmenden Einsatz von Deep-learning-Algorithmen die erforderlichen individuellen und komplexen Bewegungsmuster einer Ultraschalluntersuchung in naher Zukunft von einem Robotersystem realisiert werden und damit die Möglichkeit einer echten Automatisierung gegeben ist $[6,10]$. 


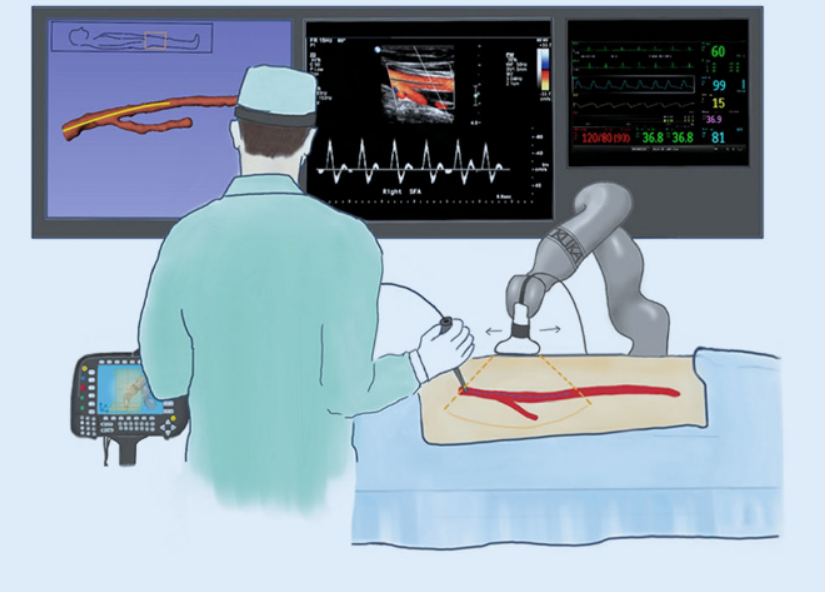

Abb. $4<$ Geplantes

klinisches Setting: Während der endovaskulären Intervention erfolgt eine automatisierte Schallkopfsteuerung. Der Arzt erhält somit eine ständige Echtzeitvisualisierung des Interventionsfokus. Mit freundlicher Genehmigung, (c) M. Kaschwich, M. Kleemann. Alle Rechte vorbehalten

\section{Schlussfolgerung und Ausblick}

Nach der klinischen Einführung und Durchsetzung der endovaskulären Verfahren in der Behandlung der peripheren arteriellen Verschlusskrankheit müssen die zukünftigen medizintechnischen Entwicklungen auf die Reduzierung von Strahlungs- und Kontrastmittelapplikation zielen. Eine bis dato wenig weiterentwickelte Form der Ultraschallbildgebung während der Intervention kann unter robotischer Assistenz, also automatisierter Schallkopfführung, zur klinischen Routine weiterentwickelt werden (• Abb. 4). Aktuell befinden sich die Entwicklungen im Stadium der technischen Machbarkeit.

Die nächsten Schritte in diesem Projekt sind:

1. Automatische Kalibration von Ultraschall- und Robotersystem

2. Vollautomatische, roboterbasierte Darstellung des gesamten Gefäßbaums im Ultraschall („Ultraschallangiogramm“), inklusive weiterer prä- und intraoperativer Daten

3. Automatische Erkennung und Verfolgung des Führungsdrahtes

4. Akquise eines Doppler-Flussbilds über die gesamte Länge der Beinarterie

5. Validierung verschiedener Verfahren zur intraarteriellen Bildgebung (intravaskulärer Ultraschall [IVUS], optische Kohärenztomographie [OCT])
Einhaltung ethischer Richtlinien

Interessenkonflikt. M. Kaschwich, F. von Haxthausen T. Aust, F. Ernst und M. Kleemann geben an, dass kein Interessenkonflikt besteht.

Für diesen Beitrag wurden von den Autoren keine Studien an Menschen oder Tieren durchgeführt. Für die aufgeführten Studien gelten die jeweils dort angegebenen ethischen Richtlinien.

Open Access. Dieser Artikel wird unter der Creative Commons Namensnennung 4.0 International Lizenz veröffentlicht, welche die Nutzung, Vervielfältigung, Bearbeitung, Verbreitung und Wiedergabe in jeglichem Medium und Format erlaubt, sofern Sie den/die ursprünglichen Autor(en) und die Quelle ordnungsgemäß nennen, einen Link zur Creative Commons Lizenz beifügen und angeben, ob Änderungen vorgenommen wurden.

Die in diesem Artikel enthaltenen Bilder und sonstige Drittmaterial unterliegen ebenfalls der genannten Creative Commons Lizenz, sofern sich aus der Abbildungslegende nichts anderes ergibt. Sofern das betreffende Material nicht unter der genannten Creative Commons Lizenz steht und die betreffende Handlung nicht nach gesetzlichen Vorschriften erlaubt ist, ist für die oben aufgeführten Weiterverwendungen des Materials die Einwilligung des jeweiligen Rechteinhabers einzuholen.

Weitere Details zur Lizenz entnehmen Sie bitte der Lizenzinformation auf http://creativecommons.org/ licenses/by/4.0/deed.de.

\section{Literatur}

1. Aboyans V, Ricco JB, Bartelink MLEL et al (2018) 2017 ESC Guidelines on the Diagnosis and Treatment of Peripheral Arterial Diseases, in collaboration with the European Society for Vascular Surgery (ESVS). Eur Heart J 39:763-816. https://doi.org/10.1093/eurheartj/ehx095

2. Ascher E, Marks NA, Hingorani AP et al (2005) Duplex-guided balloon angioplasty and subintimal dissection of infrapopliteal arteries: Early results with a new approach to avoid radiation exposure and contrast material. J Vasc Surg 42:1114-1121. https://doi.org/10.1016/j.jvs.2005.08.025

3. Ascher E, Marks NA, Hingorani AP et al (2006) Duplex-guided endovascular treatment for occlusive and stenotic lesions of the femoral-popliteal arterial segment: a comparative study in the first 253 cases. J Vasc Surg 44:1230-1237. https://doi. org/10.1016/j.jvs.2006.08.025

4. Abolmaesumi P, Salcudean SE, Zhu WH, Sirouspour MR, DiMaio SP (2002) Image-guided control of a robot for medical ultrasound. IEEE Trans Robot Autom 18(1):11-23. https://doi.org/10.1109/70. 988970

5. Bolt LJ, Krasznai AG, Sigterman TA et al (2019) Duplex-guided versus conventional percutaneous transluminal angioplasty of Iliac TASC II A and B lesion: a randomized controlled trial. Ann Vasc Surg 55:138-147. https://doi.org/10.1016/j.avsg.2018. 07.047

6. Brattain LJ, Telfer BA, Dhyani M et al (2018) Machine learning for medical ultrasound: status, methods, and future opportunities. Abdom Radiol 
43:786-799. https://doi.org/10.1007/s00261018-1517-0

7. Fusaroli P, Jenssen C, Hocke M et al (2016) EFSUMB Guidelines on Interventional Ultrasound (INVUS), Part VI. Ultraschall Med 37:E77-E99. https://doi. org/10.1055/s-0035-1553738

8. Goldsweig AM, Abbott JD, Aronow HD (2017) Physician and patient radiation exposure during endovascular procedures. Curr Treat Options Cardiovasc Med. https://doi.org/10.1007/s11936017-0507-9

9. von Haxthausen $F$, Aust $T$, Schwegmann $\mathrm{H}$ et al (2020) Visual servoing for semi-automated 2D ultrasound scanning of peripheral arteries. Automed Autom Med Eng. https://doi.org/10. 18416/AUTOMED.2020

10. Huang Q, Zhang F, Li X (2018) Machine learning in ultrasound computer-aided diagnostic systems: a survey. Biomed Res Int. https://doi.org/10.1155/ 2018/5137904

11. Janvier MA, Durand LG, Cardinal MHR et al (2008) Performance evaluation of a medical robotic 3Dultrasound imaging system. Med Image Anal 12:275-290. https://doi.org/10.1016/j.media. 2007.10.006

12. Kaschwich M, Dell A, Matysiak F et al (2020) Development of an ultrasound-capable phantom with patient-specific 3D-printed vascular anatomy to simulate peripheral endovascular interventions. Ann Anat. 2020. https://doi.org/10.1016/j.aanat. 2020.151563

13. Kaschwich M, Matysiak F, Horn M, Kleemann M (2018) Imaging procedures-possibilities for reduction of radiation in the operating room. Gefässchirurgie 23:574-579. https://doi.org/10. 1007/s00772-018-0480-y

14. Kleemann M, Böttger S, von Haxthausen F (2019) Robotics: automated procedures in vascular medicine. Gefässchirurgie 24:557-563. https:// doi.org/10.1007/s00772-019-00581-8

15. Kojcev R, Khakzar A, Fuerst B et al (2017) On the reproducibility of expert-operated and robotic ultrasound acquisitions. Int J Comput Assist Radiol Surg 12:1003-1011. https://doi.org/10. 1007/s11548-017-1561-1

16. Kuhlemann I, Ernst F (2017) A safety module for active joint limit avoidance and intuitive hand guidance of a robotic ultrasound system. Int J Comput Assist Radiol Surg 12:38-39

17. Maturo VG, Zusmer NR, Gilson AJ et al (1980) Ultrasound of the whole breast utilizing a dedicated automated breast scanner. Radiology 137:457-463. https://doi.org/10.1148/radiology. 137.2.6254110

18. Bin Mustafa AS, Ishii T, Matsunaga Y et al (2013) Human abdomen recognition using camera and force sensor in medical robot system for automatic ultrasound scan. Proc Annu Int Conf IEEE Eng Med Biol Soc EMBS, S 4855-4858 https://doi.org/10. 1109/EMBC.2013.6610635

19. Nakadate R, Solis J, Takanishi A et al (2011) Outof-plane visual servoing method for tracking the carotid artery with a robot-assisted ultrasound diagnostic system. Proc - IEEE Int Conf Robot Autom:, S 5267-5272 https://doi.org/10.1109/ ICRA.2011.5979594

20. Prasad A, Ortiz-lopez C, Khan A et al (2016) Acute kidney injury following peripheral angiography and endovascular therapy: a systematic review of the literature. Catheter Cardiovasc Interv 88:264-273. https://doi.org/10.1002/ccd.26466

21. Priester AM, Natarajan S, Culjat M (2013) Robotic ultrasound systems in medicine. IEEE Trans
Ultrason Ferroelectr Freq Control 60:507-523. https://doi.org/10.1109/TUFFC.2013.2593

22. Schäberle W (2016) Ultraschall in der Gefäßdiagnostik https://doi.org/10.1007/978-3-66247432-7

23. Seitz PK, Baumann B, Johnen W et al (2019) Development of a robot - assisted ultrasound guided radiation therapy (USgRT)

24. Sen HT, Lediju BMA, Zhang Y et al (2017) System integration and in vivo testing of a robot for ultrasound guidance and monitoring during radiotherapy. IEEE Trans Biomed Eng 64:1608-1618. https://doi.org/10.1109/TBME.2016.2612229

25. Stasi G, Ruoti EM (2015) A critical evaluation in the delivery of the ultrasound practice: the point of view of the radiologist. Ital J Med 9:5-10. https:// doi.org/10.4081/itjm.2015.502

26. Swerdlow DR, Cleary K, Wilson E et al (2017) Robotic arm-assisted sonography: review of technical developments and potential clinical applications. Am J Roentgenol 208:733-738. https://doi.org/10.2214/AJR.16.16780

27. Virga S, Zettinig O, Esposito $M$ et al (2016) Automatic force-compliant robotic Ultrasound screening of abdominal aortic aneurysms. IEEE Int Conf Intell Robot Syst, S 508-513 https://doi.org/ 10.1109/IROS.2016.7759101

28. Wang S, Housden J, Noh Y et al (2019) Roboticassisted ultrasound for fetal imaging: Evolution from single-arm to dual-arm system. Lect Notes Comput Sci (including Subser Lect Notes Artif Intell Lect Notes Bioinformatics) 11650 LNAI, S 27-38 https://doi.org/10.1007/978-3-030-25332-5_3

29. Zettinig O, Fuerst B, Kojcev R et al (2016) 2016-Toward Real-time 3D Ultrasound Registration-based Visual Servoing for Interventional Navigation.pdf. IEEE Int. Conf. Robot. Autom..

30. https://www.ema.europa.eu/en/humanregulatory/overview/medical-devices. Zugegriffen: Mai 2020
Hier steht eine Anzeige. Springer 